\title{
The word-frequency effect and incongruity perception: Methodological artifacts?
}

\author{
G. KEMPEN, ${ }^{1}$ B. HERMANS, A. KLINKUM, M. BRAND, AND F. VERHAAREN ${ }^{2}$ \\ UNIVERSITY OF NIJMEGEN, THE NETHERLANDS
}

Two experimental results often reported in support of perceptual interpretations concerning the influence of set on perception are critically examined: (a) the relation between word frequency and recognition threshold, and (b) the so-called compromise reactions between set and stimulus. Alter elimination of certain methodological artifacts (e.g., introduction of a temporal forced-choice method instead of the ascending-limits method), both phenomena disappear; the influence of set on perception appears to be wholly a matter of response bias.

Howes and Solomon (1951) and Solomon and Postman (1952) found high negative correlations between frequency and recognition threshold of English words and nonsense syllables. Bruner and Postman (1949) obtained much higher thresholds for incongruous playing cards (e.g., red spades) than for normal cards (e.g., black spades).

Since the study of Goldiamond and Hawkins (1958) the explanation of these phenomena in terms of response probabilities has been widely accepted. The latter interpretation, however, does not rule out the possibility of genuine perceptual effects resulting from implicit sets (Dember, 1960). Zajonc and Nieuwenhuyse (1964) made a further test of the response bias hypothesis by introducing very stringent recognition criteria. In this experiment the negative correlation between frequency and recognition threshold decreased with increasing stringency of the criterion used (viz., pseudorecognition, one correct identification and three successive correct identifications). Nevertheless, even in the most stringent criterion condition a very reliable effect of word frequency was found.

These results are complemented by the so-called compromise responses in the playing cards experiment by Bruner and Postman (1949). For instance, after presentation of (incongruous) red spades many Ss gave prerecognition answers such as "brown spades," "purple," "rusty black," "black with red edges," i.e., names of colors that might be considered mixtures of red and black.

These two experimental results are repeatedly quoted in support of perceptual theories concerning set-perception interactions. In the present study it is hypothesized that they must be attributed to certain methodological artifacts.

\section{EXPERIMENT 1}

The word frequency effect was supposed to disappear after the introduction of the following two procedural modifications: (a) a temporal forced-choice method for measuring recognition thresholds, and (b) a very strict recognition criterion (three successive correct identifications).

\section{Procedure}

Nonsense syllables (CVCs) of equal meaningfulness were used as stimuli. In each trial of the pretraining phase, $E$ showed one syllable to the Ss (30 undergraduate students) while asking first to spell and thereafter to pronounce it. Frequency was varied by presenting some syllables $25,10,5$, and 2 times, respectively. For each frequency there were two syllables. Counterbalancing these syllables against frequency was not considered essential, first, since their selection was restricted by the requirement of equal meaningfulness, second, because the main interest was in the expected interaction between word-frequency and method of threshold measurement. Twenty-four other CVCs of equal association value were used as context items in the test phase.

Determination of the recognition thresholds took place in two ways: the forced-choice method ( $\mathrm{FC} ; 15 \mathrm{Ss})$ and the ascending-limits method (AL; $15 \mathrm{Ss}$ ). In every trial the Ss of the FC group heard one of the eight focal syllables pronounced by $\mathrm{E}$. Immediately afterwards, four syllables were presented in a Bettendorf tachistoscope. Always one of the items was the focal one, three were context items. The intervals between the four stimuli within each trial were $2 \mathrm{sec}$; after each trial there was a 4-sec pause. Ss were instructed to indicate after each trial the serial number of the focal syllable. The recognition thresholds of the AL group were measured by means of the method of limits. In both groups the exposure times increased by steps of $.001 \mathrm{sec}$, starting at a presentation time of $.001 \mathrm{sec}$. The level of illumination of the pre- and postexposure tachistoscopic field was 2 , that of the stimulus field 5 lux, as measured at the location of the eyes. The CVCs $(3 \times 7 \mathrm{~mm})$ were typed in capitals (Pica) on white cards. The stimuli subtended a $19 \times 56 \mathrm{~min}$ visual angle. For the FC groups the recognition threshold was defined as the exposure time at which the second correct identification of three successive correct identifications occurred. For the AL group one correct response was sufficient. This lenient criterion for the $\mathrm{AL}$ group, as opposed to the strict $\mathrm{FC}$ criterion, was deliberately chosen in order to introduce ample opportunity for response bias.

\section{Results}

The product-moment correlation between recognition threshold (in milliseconds) and $\log$ frequency was found to be $r=-.97(p<.005)$ for the AL group and $r=+.19$ (n.s.) for the FC group (cf. Table 1). This means that the reported negative correlation of frequency and recognition threshold. which we were able to replicate in the AL condition, completely disappears under perfect control of response bias; if anything, it gives way to a slight positive correlation under the $\mathrm{FC}$ condition.

\section{Discussion}

The rather drastic disappearance of the word-frequency effect should be interpreted carefully. Inspection of Table 1 shows that all FC thresholds are below those of the AL group. Apparently, the FC Ss needed less information from the stimulus patterns than the AL Ss. Although, undoubtedly, also the AL Ss could rely on fragmentary perception of the stimuli, one can raise the issue whether word recognition actually

Table 1

Mean Recognition Thresholds (Milliseconds) of Four Pairs of Focal Syllables Measured by Forced-Choice and Ascending-Limits Method

\begin{tabular}{lrrrr}
\hline Method & \multicolumn{4}{c}{ Frequency } \\
& \multicolumn{1}{c}{2} & \multicolumn{1}{c}{5} & 10 & \multicolumn{1}{c}{25} \\
\hline Forced-Choice & 6.80 & 7.00 & 6.50 & 7.10 \\
Ascending-Limits & 26.70 & 23.50 & 18.30 & 11.12 \\
\hline
\end{tabular}


occurred if only very few features of the syllables (e.g., only one letter or even parts of a letter) were involved in the task. This possibility should be checked in further experimentation.

\section{EXPERIMENT 2}

In their 1949 article, Bruner and Postman mentioned that they did not succeed in having the incongruous cards printed in the "original" red and black colors of playing cards, so that they had to paint over the normal cards. The colors thus obtained were, however, not quite accurate: their red was darker and less yellow-red than on the normal cards. This induced them to paint over the normal cards as well, so that the differences in the red color between congruous and incongruous cards did no longer exist. Covering red hearts and diamonds with black paint was apparently more successful, seeing Bruner and Postman did not attempt to modify the congruous black cards.

Thus, four groups of cards were used: (a) imperfect red incongruous; (b) imperfect red congruous; (c) almost perfect black incongruous; and (d) perfect black congruous cards. For these groups the percentage of color-compromise answers was $63,32,12$, and 0 , respectively. This suggests the hypothesis that there were, in fact, two sources of incongruity: (a) color inversion and (b) imperfection of colors, and that many so-called compromise reactions were indeed correct identifications (e.g., the response "brown-red spades" after the presentation of actually dark red spades).

To test this possibility, a replication of the Bruner and Postman experiment was carried out. However, the colors were not painted over but printed in the "real" red and black of playing cards. In this situation, it was supposed, no compromise reactions would be found.

The replication was exact: the same type and number of Ss (28 nonpsychology students), the same tachistoscopic exposure times, the same playing cards, etc. (For the properties of the tachistoscope, see Experiment 1.)

The general results of this replication experiment were extremely consistent with Bruner and Postman's: equal number of dominance and disruption answers and equal effects of experience with incongruous cards were obtained.
However, there was one important difference. In the replication experiment no compromise reactions were given. Only one S responded once, "black spades with a shade of red" on presentation of red spades. But he gave an analogous response, "black hearts with a shade of red," on presentation of congruous red hearts. However, in these trials the presentation times were so short that color perception was very incomplete. Therefore, this answer cannot be considered a compromise reaction.

\section{CONCLUSION}

Inasmuch as conflicting evidence is absent in the literature, these findings, if they hold in further experiments, lead us to conclude that implicit sets have no genuine perceptual effects and that interactions between set and recognition must be interpreted wholly in terms of response bias.

\section{REFERENCES}

BRUNER, J. S., \& POSTMAN, L. On the perception of incongruity: A paradigm. Journal of Personality, 1949, 1, 206-223.

DEMBER, W. N. The psychology of perception. New York: Holt, Rinehart \& Winston, 1960.

GOLDIAMOND, I., \& HAWKINS, W. F. Vexierversuch: The log relationship between word-frequency and recognition obtained in the absence of stimulus words. Journal of Experimental Psychology, 1958, $56,457-463$.

HOWES, D. H., \& SOLOMON, R. L. Visual duration threshold.ss a function of word-probability. Journal of Experimental Psychology, $1951,41,401-410$.

SOLOMON, R. L., \& POSTMAN, L. Frequency of usage as a determinant of recognition thresholds for words. Journal of Experimental Psychology, 1952, 43, 195-201.

ZAJONC, R. B., \& NIEUWENHUYSE, B. Relationship between word frequency and recognition: Perceptual process or response bias? Journal of Experimental Psychology, 1964, 67, 276-285.

\section{NOTES}

1. Address: Psychologisch Laboratorium der Katholieke Universiteit, Nijmegen, The Netherlands.

2. The first of the two experiments reported in this article was carried out by M. Brand and F. Verhaaren, and the second one by B. Hermans and $\mathrm{A}$. Klinkum.

(Accepted for publication October 7, 1968.) 